\title{
Titel/Title: Soziale Dienstleistungen zwischen Informalisierung und Professionalisierung - oder: der schwierige Abschied vom deutschen Erbe sozialpolitischer Regulierung
}

Autor*innen/Author(s): Karin Gottschall

Veröffentlichungsversion/Published version: Verlagsversion (VoR)

Zeitschriftenartikel/Journal article

\section{Empfohlene Zitierung/Recommended citation:}

Gottschall, Karin (2008): Soziale Dienstleistungen zwischen Informalisierung und

Professionalisierung - oder: der schwierige Abschied vom deutschen Erbe sozialpolitischer Regulierung. In: Arbeit, 17 (4), 254-267.

Verfügbar unter/Available at:

(wenn vorhanden, bitte den DOI angeben/please provide the DOI if available)

Die Verlagsveröffentlichung ist verfügbar unter www.degruyter.com.

https://doi.org/10.1515/arbeit-2008-0404

Zusätzliche Informationen/Additional information:

Die Autorin kann kontaktiert werden unter:

karin.gottschall@uni-bremen.de 


\title{
Soziale Dienstleistungen zwischen Informalisierung und Professionalisierung - oder: der schwierige Abschied vom deutschen Erbe sozialpolitischer Regulierung
}

\begin{abstract}
Steigende Ansprüche an Umfang und Qualität öffentlich erbrachter sozialer Dienstleistungen stellen für Sozialstaats- und Arbeitsmarktakteure in Deutschland eine besondere Herausforderung dar. Denn Ausbildung und Beschäftigung in den sozialen Dienstleistungen sind historisch als Frauenarbeit geprägt und weichen von den Standards von Normalarbeit ab. Der Beitrag untersucht ausgehend von der eher sozial- als arbeitspolitischen Regulation sozialer Dienstleistungen, wie sich Ausbildung und Beschäftigung in den Gesundheits-, Erziehungs- und Sozialberufen entwickelt haben und inwieweit jüngere Reformen und Re-Regulierungen zu einer nachhaltigen Berufsförmigkeit beitragen.
\end{abstract}

\section{$1 \quad$ Einleitung}

Wie Kranke behandelt, wie Kinder betreut, wie Schüler unterrichtet und wie alte Menschen gepflegt werden, ist in jüngerer Zeit zunehmend Gegenstand gesellschaftspolitischer Debatten. Damit erlangen Tätigkeiten und Arbeitsstätten, Beschäftigte, soziale Gruppen und Lebensphasen öffentliche Aufmerksamkeit, die im ,Modell Deutschland' mit seinem industriegesellschaftlichen produktiven Kern bisher eher eine Nebenrolle gespielt haben - als Dienstleistungen, die zwar Lebensqualität aber kaum die gesamtwirtschaftliche Produktivität erhöhen und als Frauenarbeitsbereiche, die oft nicht an den Standards von Normalarbeit gemessen werden. Neu ist, dass Fragen der Qualität von Bildung und Erziehung, der Finanzierung und Organisation von Pflege unter dem Druck von globalem Wettbewerb und dem Einfluss internationaler Organisationen wie OECD und EU nicht mehr vorrangig als sozialpolitische sondern vor allem auch als ökonomische Herausforderung behandelt werden. Die jüngsten Proteste von Krankenhausärzten und Krankenpflegepersonal zeigen darüber hinaus, dass es hier mit Themen wie Einkommen, Arbeitszeit und Arbeitsplatzsicherung nun auch um genuin arbeitspolitische Forderungen_geht. Damit sind soziale Dienstleistungen, die historisch gesehen einen langen Weg aus der privaten Sphäre in den öffentlichen Raum zurückgelegt haben, nicht nur im Hinblick auf den Umfang der Beschäftigung sondern auch im Hinblick auf ihr soziales Konfliktpotenzial in der Mitte der Gesellschaft angekommen.

Offensichtlich lassen sich die angesprochenen Probleme und Frontstellungen jedoch nicht einfach nach dem Muster industriegesellschaftlicher Entwicklungsdynamik und Konfliktbearbeitung lösen. Dies hängt auch mit den Besonderheiten der ,Arbeit am und mit Menschen' zusammen, die insbesondere als fürsorgliche Tätigkeit sowohl aus der 
Sicht der Fürsorgenden wie auch der Empfänger zuallererst auch menschliche Beziehung ist, deren Vermarktlichung nur unter spezifischen Bedingungen wünschenswert erscheint. In kapitalistischen Marktökonomien dienen sozialen Dienstleistungen der Herstellung und dem Erhalt der Lohnarbeitskraft und unterliegen historisch eher sozialpolitischer als arbeitspolitischer Regulation. Dies ist im deutschen Produktions- und Sozialmodell besonders ausgeprägt, wo diese Tätigkeiten in einem Kontinuum von privater Sorgearbeit, ehrenamtlicher Tätigkeit und Berufsarbeit institutionalisiert und kulturell als Frauenarbeit normiert sind. Als ,verberuflichte’ Tätigkeit weicht Sorgearbeit von der Regulation und den Standards des Normalverhältnisses vielfach ab, wie sich in der starken Rolle öffentlicher und quasi-öffentlicher Arbeitgeber, besonderen Ausbildungsformen und Beschäftigungsstrukturen zeigt. War die Komplementarität von arbeits- und sozialpolitischer Regulation in Zeiten ökonomischer Prosperität und Sozialstaatsausbau funktional, so gerät unter postindustriellen Bedingungen neben der arbeitspolitischen Steuerung auch die sozialpolitisch geprägte Steuerung der Gesundheits-, Sozial- und Erziehungsberufe unter Druck: durch erhöhte Nachfrage nach diesen Dienstleistungen, neue Qualitätsansprüche, veränderte Erwerbsmuster und Probleme öffentlicher Finanzierung. Wie ein Blick auf andere Länder zeigt, sind unterschiedliche Antworten auf diese Probleme möglich; in Deutschland stellt sich, so die These des Beitrags, vor allem die Sicherung von Berufsförmigkeit als Herausforderung dar.

Im Folgenden werden unter sozialen Dienstleistungen Erziehungs-, Sozial- und Gesundheitsberufe verstanden und unter funktionalen Gesichtspunkten von kommerziellen Dienstleistungen abgegrenzt. Kennzeichnend für soziale Dienstleistungen ist, dass sie im weitesten Sinn auf die Reproduktion der Arbeitskraft ausgerichtet sind und in Deutschland bisher überwiegend in öffentlichen und gemeinnützigen Einrichtungen erbracht werden; im Unterschied zu kommerziellen Dienstleistungen, die u. a Tätigkeiten in Handel, Handwerk und Finanzsektor umfassen, unterliegen sie nur begrenzt der Marktlogik (Berger/Offe 1984) ${ }^{1}$. Zunächst werden die sozialen Dienstleistungsberufe im Hinblick auf ihre Stellung im deutschen Berufsbildungssystem und ihre spezifische sozialpolitische Regulierung betrachtet (2). Ausgehend von dieser Bestandsaufnahme werden aktuelle Herausforderungen im Bereich sozialer Dienstleistungen thematisiert (3) und anschließend mit jüngeren Entwicklungsdynamiken in Ausbildung und Beschäftigung wie auch politischen Reformen konfrontiert (4). Beschäftigungsstrukturelle Polarisierung, Akademisierung und anhaltende Regulierungsdefizite deuten auf einen komplexen und langsamen Wandel hin, der, so das Fazit (5), auch mit Blick auf Entwicklungen in den europäischen Nachbarländern weiteren Handlungsbedarf signalisiert.

1 Berger/Offe definieren Dienstleistungen in Abgrenzung zu Industriearbeit nach ihrer Funktion im Reproduktionsprozess kapitalistischer Gesellschaften als ,Instandhaltung von etwas'. Die quantitative Entwicklung dieses Sektors erklärt sich demnach nicht in erster Linie aus der Dynamik technischen Fortschritts sondern der Summe strategischer Reaktionen politischer Akteure auf die strukturellen Risiken einer Gesellschaft. Sie unterscheiden neben den bereits genannten kommerziellen Dienstleistungen, wo Ertrags- und Kostenkriterien wie in der materiellen Warenproduktion zur Anwendung gelangen und den öffentlichen Dienstleistungen, wo Kosten- und Ertragskriterien kaum Anwendung finden, noch unternehmensinterne Dienstleistungen, die durchaus Kosten-, aber nicht unbedingt auch Ertragskriterien unterliegen (Berger/Offe 1984, 233). Zum Ertrag dieses Verständnisses vgl. auch Gottschall 2001. 


\section{Erziehungs-, Sozial- und Gesundheitsberufe als Sonderfall? Zur Differenz und Komplementarität arbeits- und sozialpolitischer Regulierung im deutschen Produktions- und Sozialmodell}

Ökonomische Stärke und soziale Integrationskraft des, deutschen Modells' werden im internationalen Vergleich in der Regel mit dem Prinzip der Beruflichkeit in Verbindung gebracht. Der Beruf als Institution gilt als zentraler Bestandteil der spezifischen Verfasstheit des deutschen Kapitalismus, hervorgebracht durch ein besonderes Ausbildungsmodell, das duale System, gestützt durch eine qualifikationsorientierte industrielle Arbeitsorganisation mit betrieblichen Aufstiegswegen sowie eine Strukturierung von Erwerbsarbeit als Lebensberuf. Kennzeichnend für die Regulierung der Ausbildung (wie der Beschäftigungsverhältnisse) ist ein korporatistisches Regulationsregime, das, anders als reine Marktmodelle wie in den USA oder Großbritannien oder etatistisch-bürokratische Schulausbildungsmodelle wie in Frankreich, eine gemeinsame Verantwortung von Arbeitgebern, Arbeitnehmern und staatlichen Körperschaften beinhaltet. So ist das duale Ausbildungssystem seit 1969 durch das Berufsausbildungsgesetz bundeseinheitlich geregelt; über Veränderungen von Ausbildungsinhalten entscheiden tripartistische Institutionen und die entsprechenden Ausbildungsverhältnisse sind bisher überwiegend in tarifvertragliche Entlohnung und betriebliche Mitbestimmung eingebunden. Die spezifische Praxisorientierung der Ausbildung, historisch dem handwerklichen Meister-Lehrlingsmodell entlehnt, wie auch die Einbindung der Arbeitgeber und Betriebe in die Ausbildungsorganisation ermöglichen im Bereich der mittleren Qualifikationen in der Industrie und Teilen der kommerziellen Dienstleistungen wie Banken und Versicherungen einen vergleichsweise friktionslosen Übergang von Ausbildung in Beschäftigung und generieren, da auch Entlohnung, Arbeitsbedingungen und Weiterqualifizierungen an den Beruf gebunden sind, eine relativ hohe Stabilität beruflicher Laufbahnen (Baethge 1999). ${ }^{2}$ Als schützende Hülle dieser Form von Beruflichkeit fungiert ein Normalarbeitsverhältnis, das auf Dauer und als Vollzeitarbeit angelegt ist und typische Marktrisiken abhängiger Arbeit wie Arbeitslosigkeit, Krankheit und Alter durch statusorientierte soziale Sicherungen abfedert. Nicht zufällig verkörpert der männliche Facharbeiter aus den industriellen Kernsektoren den Prototyp dieses ,verberuflichten Arbeitnehmers' (Voß/Pongratz 1998).

\section{Die Semi-Professionalität sozialer Dienstleistungsberufe}

Dienstleistungstätigkeiten in den Bereichen Erziehung, Bildung und Gesundheit auf mittlerem Qualifikationsniveau (d.h. unterhalb der akademischen Professionen) folgen freilich nicht ohne weiteres diesem Muster von Beruflichkeit. Dies gilt für die Ausbildung ebenso wie die Strukturierung von Beschäftigung. So sind die Ausbildungen für Tätigkeiten im Bereich von Kranken- und Altenpflege, Kinderbetreuung und Sozialarbeit nicht im Rahmen des dualen Systems sondern überwiegend als vollzeitschulische Ausbildungen organisiert, unterliegen damit der Kulturhoheit der Länder und gewährleisten angesichts der föderalen Heterogenität nur begrenzt Qualifikationsschutz und Transferierbarkeit. Historisch tradiert fungieren Wohlfahrtsverbände bis heute häufig als Träger der Ausbildungseinrichtungen und zugleich als Arbeitgeber, die gleichzeitig auch über den Bedarf

2 Dies gilt am ehesten für die industriebezogenen Ausbildungsberufe, während die (weiblich dominierten) Ausbildungen und Berufstätigkeiten in Einzelhandel, Gastronomie und Friseurgewerbe eher durch geringe Verdienstmöglichkeiten, Teilzeitarbeit und Fristigkeit gekennzeichnet sind. 
an Fachpersonal, Personaleinsatz und Fachprofile entscheiden. Weitere Unterschiede zur dualen Ausbildung liegen im abhängigen Status der Auszubildenden als Schüler/innen und der Notwendigkeit die Ausbildung privat zu finanzieren. Die formalen Eintrittsqualifikationen sind mit mittlerer Reife und Abitur in der Tendenz höher als im dualen System, gleichzeitig setzt sich dieser Qualifikationsvorsprung im Allgemeinbildungsniveau jedoch nicht in höhere Arbeitsmarkterträge um. Dies hängt zunächst damit zusammen, dass die Übergänge von der Ausbildung in Beschäftigung anders als bei industriellen Tätigkeiten bei sozialen Dienstleistungen angesichts der fehlenden Standardisierung der Qualifikation $^{3}$ weniger gesichert sind. Darüber hinaus sind soziale Dienstleistungen in Deutschland historisch als Semi-Professionen und damit , beruflich' defizitär strukturiert: So fehlen bisher innerbetrieblich geregelte Aufstiegswege (als Erzieherin in einer Kindertagesstätte etwa steht allenfalls ein Weg zur Leiterin der Einrichtung offen). Ebenso fehlen Möglichkeiten wissenschaftlicher Weiterbildung, da das Ausbildungspersonal in diesem Bereich in der Vergangenheit typischerweise selbst nicht akademisch qualifiziert war ${ }^{4}$. Kennzeichnend etwa im Bereich der Krankenpflege ist in Deutschland vielmehr ein hierarchisches Verhältnis der semi-professionellen Krankenpflege zu der durch ein akademisches Ausbildungsniveau deutlich abgegrenzten medizinischen Profession: In dieser Konstellation wird Pflege im Unterschied zur Medizin als nicht-wissenschaftlich basiert und als Zuarbeit definiert. In anglo-amerikanischen Ländern hingegen gelten Pflegekräfte und Ärzte eher als gleichrangig, nicht zuletzt da die Ausbildung für Pflegetätigkeiten durch BA-Studiengänge wesentlich stärker durch anerkannte fachwissenschaftliche Expertise gekennzeichnet ist. Schließlich sind auch die Beschäftigungsverhältnisse in Krankenhäusern, Altenheimen und Kindergärten nur bedingt als Normalarbeitsverhältnis ausgestaltet, wie die hohe Verbreitung von Teilzeitarbeit und eine verbreitete Diskontinuität von Berufslaufbahnen zeigen (Krüger 1995, 2003).

Ausbildung und Beschäftigung im Bereich sozialer Dienstleistungen sind damit im Vergleich zu industriellem Sektor und kommerziellen Dienstleistungen weniger durch (korporatistische) arbeitspolitische als vielmehr durch sozialpolitische Regulierung gekennzeichnet: Nicht nur unterliegt die Ausbildung der uneinheitlichen Governance des föderal-staatlichen Bildungswesens, auch Umfang und Struktur der Arbeitsplätze sind weniger von der Marktdynamik als vielmehr dem Ausbau und der Organisation des Wohlfahrtsstaates abhängig und damit politisch gerahmt. Dabei wirken sich zwei Prinzipen deutscher Sozialstaatlichkeit für die Ausbildung von Beruflichkeit nachteilig aus: Durch das Subsidiaritätsprinzip, das staatliche Leistungen erst nach Ausschöpfung von Selbsthilfe in Familie und lokalen Bezügen vorsieht, erfolgen staatliche Sozialleistungen eher in Form von materiellen Transfers als von Dienstleistungen. Letztere sind insbesondere im Bereich von Kinderbetreuung und Primarschulwesen im europäischen Vergleich eher gering ausgebaut und durch eine Halbtagskultur geprägt, die eine Ausgestaltung auch der Beschäftigungsverhältnisse als Teilzeitarbeit begünstigt (Gottschall/Hagemann 2002). Darüber hinaus werden soziale Dienstleistungen nicht nur durch die öffentliche Hand sondern überwiegend durch die (häufig konfessionellen) Wohlfahrtsverbände erbracht, die bis heute entsprechend ihrem Selbstverständnis durch eine Mischung von

3 Zwar ist die Berufsbezeichnung geschützt, gleichwohl können die Ausbildungsinhalte träger- und länderspezifisch variieren (Krüger 2003, 502)

4 Anschlüsse an zweite Bildungswege im berufsbildenden Bereich fehlen auch, weil im Schulberufssystem, anders als im dualen System, häufig die für solche Weiterbildungen notwendigen allgemeinbildenden Fächer nicht unterrichtet werden. Dies trifft insbesondere weibliche Auszubildende, die nur über die mittlere Reife verfügen (Krüger 2003, 503) 
professioneller und ehrenamtlicher Arbeit gekennzeichnet sind (Evers/Sachße 2003). Wie sozialhistorische Studien zur Entwicklung von Krankenpflege und Sozialarbeit zeigen, hat die religiöse und sozialmoralische Fundierung dieser Tätigkeiten, die Idealen des selbstlosen Dienstes, der Aufopferung und Freiwilligkeit folgt, wesentlich dazu beigetragen, dass sich die beteiligten Akteure historisch eher spät und nur begrenzt für eine, allgemeinen Standards verpflichtete Ausbildung und geldwerte Anerkennung engagiert haben $^{5}$ - ganz anders als die Professionen in den Bereichen Bildung und Gesundheit, die, wie die Beispiele Gymnasiallehrer und Arzt zeigen, die ethisch-moralische Aufladung ihrer Tätigkeit mit staatlicher Unterstützung in Deutschland erfolgreich in ökonomisches Kapital, Monopolstellung und soziale Statusgewinne umzumünzen wussten. Die historische Langzeitwirkung dieser Spaltung in Professionen und Semi-Professionen zeigt sich in Deutschland in der Statusdifferenz von Arzt und Krankenschwester/pfleger ebenso wie in dem Verhältnis von Lehrer und Erzieher/in oder der verspäteten Aufnahme der ,Kleinkindschullehrer' in die mit universitärer Ausbildung und Beamtenprivilegien ausgestattete Lehrerschaft (vgl. mit Nachweisen Gottschall 2004).

\section{Geschlecht und Berufsstruktur}

Schließlich geht die Strukturdifferenz von arbeitspolitischer Regulierung von industrieller Arbeit und kommerziellen Dienstleistungen einerseits und sozialpolitischer Regulierung von sozialen Dienstleistungsberufen andererseits auch mit einer umfassenden geschlechtsspezifischen Arbeitsteilung einher. Historisch sollte die Ausbildung für Industrie- und Handwerksberufe männliche Jugendliche aus der Arbeiterschaft auf die Rolle des männlichen Familienernährers, die Ausbildung für die Sozialberufe hingegen junge Frauen aus bürgerlichen Schichten auf die Tätigkeit als Hausfrau und Mutter, ehrenamtliches Engagement oder, im Fall des Fehlens eines Familienernährers, auf eine geschlechterkulturell angemessene Erwerbstätigkeit vorbereiten. Tatsächlich konnte in den Nachkriegsjahrzehnten in der alten Bundesrepublik mit starken Industriegewerkschaften und unterstützt durch Sozialstaatsausbau für die Mehrzahl der männlichen Beschäftigten ein Familienlohn durchgesetzt werden. So gewann auch in der Arbeiterschicht das ursprünglich bürgerliche Familienmodell eines männlichen Familienernährers und einer weiblichen Hausfrau und Mutter, deren Erwerbsbeteiligung nur ,Zuverdienstcharakter' hat, praktisch an Bedeutung (Hinrichs 1996). Diese geschlechtsspezifische Rollenteilung in der Familie geht ungeachtet gestiegener Erwerbsbeteilung und höherer Qualifikationsniveaus von Frauen bis heute mit einer hohen geschlechtsspezifischen Arbeitsmarktsegregation einher: Soziale Dienstleistungen sind nach wie vor Frauendomänen und industriell- technische Tätigkeiten männerdominiert; selbst in der DDR ist diese Geschlechtsspezifik der Erwerbsstruktur nicht erodiert (Achatz 2005). Zusammenfassend kann festgehalten werden, dass die geschilderten unterschiedlichen Regulierungslogiken in Ausbildung und Beschäftigung nicht einfach nebeneinander sondern in einem besonderen Verweisungszusammenhang stehen: Sie funktionieren auf dem Arbeitsmarkt als differente Logiken, wobei die Logik des Normalarbeitsverhältnisses dominiert; beide Logiken zusammen verhalten sich komplementär zu einem Familienmodell, das über die Rolle der Hausfrau und Mutter in größerem Umfang Sorgearbeit einschließt.

5 Vgl. zur sozialen Arbeit Paulini (2007), zu Erziehungsberufen/Kinderbetreuung Nagel (2000), zu den Gesundheitsberufen Sewtz (2006). 


\section{Aktuelle Herausforderungen}

Die geschilderte Ausgangssituation: ein eher geringes Angebot an sozialen Dienstleistungen insbesondere in der Kinderbetreuung und im Bereich der Pflege aufgrund hoher familialer Solidarität wie auch die Semiprofessionalität der entsprechenden Berufe beruhte in Westdeutschland bis in die achtziger Jahre des letzten Jahrhunderts durchaus auf einem gesellschaftlichen und politischen Konsens, denn die Mehrzahl der Familien folgte einem Erwerbsmuster, in dem Frauen gar nicht oder aber nur phasenweise und in Teilzeit erwerbstätig waren (SOFI u.a. 2005, 17 ff.). Dieser Konsens ist inzwischen aus unterschiedlichen Gründen im Schwinden begriffen. So ist nicht nur das Ausbildungsniveau von Frauen deutlich gestiegen, so dass weibliche Jugendliche heute über eine höhere Allgemeinbildung als männliche Jugendliche verfügen und die Hälfte der erfolgreichen Universitätsabsolventen stellen (Autorengruppe Bildungsberichterstattung 2008, 90 ff., 133). Auch die Erwerbsbeteiligung von Müttern hat sich sukzessive erhöht und die Erwerbsverläufe insbesondere von qualifizierten Frauen sind kontinuierlicher geworden. ${ }^{6}$ Neben das weit verbreitete, durch Teilzeiterwerbstätigkeit von Frauen gekennzeichnete, modernisierte Ernährermodell sind nicht zuletzt im Zuge der Wiedervereinigung weitere Erwerbsmuster getreten (Dingeldey 2006). Gleichzeitig unterminieren eine lang anhaltende strukturelle Arbeitslosigkeit und zunehmend flexibilisierte Arbeitsmärkte den Familienernährerlohn und männliche Erwerbsarbeitskontinuität. ${ }^{7}$ Schließlich ist auch die politische und gesellschaftliche Akzeptanz für außerhäusliche Kleinkindbetreuung und für Ganztagsschulen gestiegen, wie man an den bundesweiten Ausbauprogrammen für Kinderbetreuung (vgl. die Einführung eines Rechtanspruches auf einen Halbtagskindergartenplatz 1999 und das Tagesbetreuungsförderungsgesetz 2005) und den länderspezifischen Initiativen für Schulstrukturreformen sehen kann. Dabei spielen nicht nur die Zunahme von Einelternfamilien, erhöhte Frauenerwerbstätigkeit, sinkende Geburtenraten und die hohe Akzeptanz von öffentlicher Erziehung in der ostdeutschen Bevölkerung eine Rolle. Vielmehr zeigen das schlechte Abschneiden der Bundesrepublik in der PISA-Studie und die hohen Quoten von Jugendlichen eines Abschlussjahrganges, die keinen Hauptschulabschluss erlangen und/oder sich in Maßnahmeausbildungswarteschleifen befinden, ebenso wie die im OECD-Ländervergleich hohe soziale Selektivität von Kindergarten und Schule in Deutschland, dass öffentliche Erziehung und Bildung in der gegenwärtigen Verfassung erhebliche Defizite aufweisen (vgl.OECD 2007; Baethge/Solga/Wieck 2007, 20 ff.). Die Kompensation dieser Defizite wie auch die mit einer alternden Bevölkerung steigenden Pflegebedarfe lassen sich aller Voraussicht nach in Zukunft nicht allein im Familienverbund und über weibliche Angehörige bewältigen. Bereits gegenwärtig sind circa zwei Millionen Menschen in der Bundesrepublik pflegebedürftig bzw. Leistungsempfänger der Pflegeversicherung; folgt man dem siebten Familienbericht, so wird allein bis 2020 ein

6 Die Erwerbstätigkeitsquote von Frauen ist von 1996 bis 2007 von 55,4\% auf 64,0\% gestiegen und hat sich damit der Erwerbstätigenquote der Männer, die im selben Zeitraum von 72,7 \% auf 74,7\% angestiegen ist, weiter angenähert. Der Bedeutungszuwachs des modernisierten Ernährermodells zeigt sich vor allem darin, dass die Ausweitung der Frauenerwerbstätigkeit mit einem Anstieg der Teilzeitbeschäftigung bei Frauen von 33,3\% (1996) auf 45,4 \% (2006) einherging und Teilzeitbeschäftigung insbesondere bei Müttern und verheirateten Frauen weit verbreitet ist (vgl. Statistisches Bundesamt, Arbeitskräfteerhebung der EU, abgerufen am 17.10.2008 über Bundeszentrale für politische Bildung: http://www.bpb.de/wissen/37OUAU,0,0,Die_soziale_Situation_in_Deutschland.html; ergänzend: Bothfeld u.a. 2005).

7 So waren 2004 ein Fünftel aller Beschäftigen, darunter 31\% Männer Niedriglohnbezieher, wobei die Hälfte aller Niedriglohnbezieher Vollzeit erwerbstätig ist (Kalina/Weinkopf 2006). 
Anstieg auf drei Millionen erwartet (Backes/Amrhein/Wolfinger 2008). Insgesamt steht also einem schrumpfenden Potenzial an familialen und ehrenamtlichen Leistungen für Kinder und Jugendliche, Alte und Kranke ein quantitativ und qualitativ gestiegener Bedarf an entsprechenden öffentlichen und privatwirtschaftlichen sozialen Dienstleistungen gegenüber. Hier kommt es nicht nur zu Ungleichgewichten in Arbeitsangebot und -nachfrage, wie etwa schon länger die Rekrutierung von Ausländerinnen für die Kranken- und Altenpflege zeigt. Offensichtlich zu Tage treten nunmehr auch erhebliche Kompetenzdefizite, etwa im Bereich der Frühpädagogik und Gerontologie, die eng mit der unzureichenden Professionalisierung in den entsprechenden Berufsfeldern zusammenhängen.

\section{Widersprüchliche Entwicklungsdynamiken in Ausbildung, Beschäftigung und Regulierung}

Ein genauerer Blick auf die Entwicklungen der sozialen Dienstleistungsberufe in Ausbildung und Arbeitsmarkt sowie auf die politischen Maßnahmen in diesem Feld in den letzten beiden Jahrzehnten ergibt ein widersprüchliches Bild von quantitativer Expansion, beschäftigungsstruktureller Polarisierung und zögerlicher Professionalisierung.

Zur Expansion des Dienstleistungssektors in den letzten Jahrzehnten haben nicht nur kommerzielle sondern vor allem auch nicht-kommerzielle Tätigkeiten beigetragen. Dabei handelt es sich um einen EU-weiten Trend, der in Deutschland auch in den jüngeren Phasen stagnierender Gesamtbeschäftigung gilt und zu einer überdurchschnittlichen Anteilssteigerung des Bereichs an der Gesamtbeschäftigung beiträgt. Während der Anteil der Gesundheits- und Sozialdienste ${ }^{8}$ an der Gesamtbeschäftigung in der EU-15 von 1995 bis 2003 von $9 \%$ auf $10 \%$ angestiegen ist, stieg er in Deutschland von $9 \%$ auf $11 \%$. Auch die Beschäftigungsquote im Gesundheits- und Sozialsektor in Deutschland lag 2003 mit $7 \%$ über dem EU-25 Durchschnitt von 6\%, wobei freilich in Großbritannien (8\%), den Niederlanden (10\%) und den nordeuropäischen Ländern (Schweden 12\%) ein noch höherer Anteil der Erwerbsbevölkerung in diesen Bereichen tätig ist (Europäische Kommission 2006, 21 f.). Weiterhin ist dieser Bereich in der EU-25 mit einem Frauenanteil von 78\% nach wie vor frauendominiert und weist zugleich generell eine Tendenz zur Beschäftigung von Hochqualifizierten auf, deren Anteil 2004 mit 30\% rund 10\% über dem Anteil in der Gesamtwirtschaft lag (ebd.). Beschäftigungsprognosen gehen für Deutschland von einem weiter steigenden Fachkräftebedarf insbesondere im Bereich der Rehabilitation und der Pflege aus.

\section{Beschäftigungsentwicklung nach Berufen}

Betrachtet man nun die Beschäftigtenentwicklung nach Berufsgruppen und Qualifikation in Deutschland in jüngerer Zeit unter Berücksichtigung von Arbeitszeiten so stellt sich freilich der generelle Wachstumstrend differenzierter dar. ${ }^{9}$ Zum einen weisen nicht alle

8 Sozialdienstleistungen umfassen hier alle Aufgaben im öffentlichen Interesse, d.h. auch die Sozialverwaltungen und den öffentlichen Wohnungssektor. Nicht berücksichtigt sind allgemeine Bildung und Berufsausbildung (vgl. Europäische Kommission 2006, 7)

9 Im Folgenden werden für die Gesundheitsberufe die Bereiche Kranken- und Altenpflege mit drei- und einjährigen vollzeitschulischen Ausbildungen sowie ergänzend die Arzthelfer/innen in dualer und schulischer Ausbildung berücksichtigt, für den Bereich Erziehung die vollzeitschulische Erzieher/innenausbildung und für die Sozialberufe die Fachschul- und Fachhochschulausbildungen für Sozialarbeit und Sozialpädagogik. 
Berufsgruppen Wachstum auf. So ist (nach Mikrozensusdaten) die Beschäftigung von Krankenschwestern/-pflegern zwischen 2003 und 2006 um $3 \%$ auf 788.000 zurückgegangen, während die Beschäftigung von Altenpfleger/innen um 14\% auf 478.000 angestiegen ist und die der Arzthelfer/innen praktisch stagniert. Gleichzeitig ist bei den Pflegeberufen ein deutlicher Trend zu Teilzeitarbeit unter 21 Stunden zu erkennen. So hat in der Krankenpflege bei insgesamt abnehmender Beschäftigung die Zahl der Teilzeitbeschäftigten unter 21 Stunden um 6\% zugenommen und in der Altenpflege geht das Wachstum überproportional auf Teilzeitarbeit zurück: Während die Beschäftigung über 31 Stunden nur um 2\% gestiegen ist, hat die Zahl der Erwerbstätigen, die weniger als 21 Stunden arbeiten um 34\% zugenommen (StBA 2005, 2008). Die Altenpflege hat sich damit innerhalb kurzer Zeit zu einem Teilzeitberufsfeld ausgebildet: So waren $2005 \mathrm{mehr}$ als zwei Drittel des Personals in der ambulanten und mehr als die Hälfte des Personals in der stationären Pflege Teilzeit-Beschäftigte (Backes/Amrhein/Wolfinger 2008, 49). Auch die Berufsgruppe der Erzieher/innen, die zwischen 2003 und 2006 um 6\% auf 468.000 angewachsen ist, weist Rückgänge bei der Vollzeit- und starke Zunahmen (plus 11\%) bei der Teilzeitbeschäftigung auf (StBA 2005, 2008). ${ }^{10}$ Entsprechend ist auch der Anteil der Erwerbstätigen in diesen Berufen, die weniger als 700 Euro netto verdienen, zwischen 2003 und 2006 deutlich angestiegen, z. B. bei den Erzieher/innen um 19\% und bei den Altenpfleger/innen um 20\%. Dieser Trend zu geringfügiger Teilzeitarbeit stimmt zwar mit der generellen Beschäftigtenentwicklung insbesondere bei Frauen in den letzten Jahren überein; allerdings ist das Wachstum von Teilzeitbeschäftigung in den angeführten Berufen überdurchschnittlich (StBA 2005, 2008). Eine branchenspezifische Betrachtung der Minijob-Entwicklung bestätigt diesen Trend: So ist die Zahl der gering entlohnten Beschäftigten im Wirtschaftszweig Gesundheits-, Veterinär- und Sozialwesen von 2003 bis 2006 (jeweils IV. Quartal) um 11\% gestiegen, während der Gesamtanstieg 6\% betrug. Dabei liegt das Gesundheitswesen 2007 mit einer Gesamtzahl von 410.000 geringfügig Entlohnten an zweiter Stelle der Einzelbranchen mit einer besonders hohen Zahl geringfügig Entlohnter (Bundesknappschaft 2003; Deutsche Rentenversicherung 2007).

\section{Entwicklung der Ausbildungen}

Ein Blick auf die Ausbildungsverhältnisse bestätigt vor allem für die Gesundheitsberufe den Trend zur Verschiebung zugunsten der Altenpflege und zeigt korrespondierend zur Ausweitung von atypischen Arbeitsformen eine Ausweitung von Kurzzeitausbildungen. So haben die Schülerzahlen in den Ausbildungsberufen zum Gesundheits- und Krankenschwester/-pfleger von 1998/99 bis 2006/07 um 20\% abgenommen, die Ausbildungen der Altenpflege hingegen um 30\% zugenommen. Ebenfalls Zuwächse gibt es in den quantitativ weniger bedeutsamen Berufen Physiotherapie (plus 13\%), Rettungsassistenz (plus 140\%) und der schulischen Ausbildung ,Arzthelfer/in' (plus 15\%). ${ }^{11}$ Gleichzeitig weist die Pflegeausbildung neben der Verschiebung zugunsten der Altenpflege eine Expansion

Bezogen auf die Erwerbstätigen mit entsprechendem beruflichen Abschluss handelt es sich konservativ geschätzt um mehr als 2,3 Mio. Beschäftigte; unter Berücksichtigung aller Erwerbstätigen in diesen Feldern ist die Gruppe deutlich größer.

10 Ähnliches gilt in längerfristiger Perspektive für die Berufsgruppen, Sozialarbeiter/innen/Sozialpfleger/innen', wo sich der Anteil der sozialversicherungspflichtigen Teilzeitbeschäftigen im Zuge der Expansion von 24\% im Jahr 1993 auf einen Anteil von 42\% im Jahr 2007 erhöht hat (Beschäftigtenstatistik der BA. Berufe im Spiegel der Statistik, abrufbar unter http://www. pallas.iab.de/bisds/berufe.htm).

11 Diese Ausbildung existiert auch im dualen System, wo die Mehrzahl der Beschäftigten ausgebildet wird. 
der weniger qualifizierten ein- bis zweijährigen Ausbildungen auf. So hat in der Krankenpflege parallel zum Abbau der dreijährigen Ausbildung die Zahl der Schüler/innen in der Krankenpflegehilfeausbildung um 11\% zugenommen. In der Altenpflege wiederum geht die deutliche Expansion in hohem Maß auf die Ausbildung zum ,Altenpflegehelfer/ in' zurück, die ausgehend von geringer Bedeutung in den letzten Jahren einen rasanten Zuwachs verzeichnet (von 143 Schüler/innen 1998/99 auf 6.251 in 2006/07). Im Zuge dieser Entwicklung bleibt die Frauendominanz erhalten, auch wenn Männer, die bisher nur in der Rettungsassistenz dominiert haben, inzwischen auch in den traditionell weiblich dominierten Ausbildungen der Gesundheitsberufe stärker präsent sind: In der Krankenpflegehilfe etwa ist der Männeranteil von 19\% (1998/99) auf 26\% (2006/07) gestiegen (StBA 2007, 2003, 1999).

Auch bei den Erziehungs- und Sozialberufen sind die Ausbildungszahlen angestiegen, allerdings ergibt sich hier neben einem Anstieg der Kurzzeitausbildungen in der Kinderpflege sowohl eine Ausweitung der dreijährigen fachschulischen Erzieherausbildungen wie auch der höherwertigen (sozialpädagogischen) Fachhochschulausbildungen (ebd.).

Der sich insgesamt abzeichnende Trend einer quantitativen Expansion sozialer Dienstleistungen bei gleichzeitiger qualifikatorischer und beschäftigungsstruktureller Polarisierung tritt noch deutlicher zu Tage, wenn man - über das Schulberufssystem hinaus - die allgemeinen Strukturveränderungen im Ausbildungssystem unter Einschluss der höheren Bildung in den Blick nimmt. Hier zeigt sich zum einen, dass sich der Stellenwert des Berufsausbildungssystems durch Tertiarisierung und Verwissenschaftlichung der Produktion zugunsten akademischer Ausbildung verringert: so ist die Studienanfängerquote in Deutschland zwischen 1995 und 2007 um fast 10\% auf 36,6\% gestiegen (Autorengruppe Bildungsberichterstattung 2008, 292: Tab. F1-1A) Ein weiterer Bedeutungsverlust zeigt sich in dem deutlich gestiegenen Anteil Jugendlicher, die den Eintritt in das Berufsausbildungssystem nicht schaffen und , unterhalb' in das Übergangssystem einmünden (Baethge/ Solga/Wieck 2007, 22). Zum anderen verändert sich die Struktur des Berufsausbildungssystems selbst: Bei anhaltender Dominanz der dualen Ausbildung (Anteil innerhalb des Berufsausbildungssystems circa 2/3) gewinnen die Schulberufsausbildungen (Anteil circa 1/3) an Bedeutung, die zwischen 1996 und 2006 ihren Anteil an der Gesamtgruppe der Ausbildungsabsolventen um 8\% steigern konnten, während die duale Ausbildung um 5\% abgenommen hat (Autorengruppe Bildungsberichterstattung 2008, 112). Dabei geht die absolute und anteilige Steigerung der vollzeitschulischen Ausbildungen wesentlich auch auf Schulen des Gesundheitswesens zurück (Autorengruppe Bildungsberichterstattung 2008, 287: Abb. E5-5A, 112: Abb.E5-2A).

\section{Widersprüchliche Semiprofessionalität: Offen nach unten und oben}

Für die Gesundheits-, Sozial- und Erziehungsberufe bedeutet diese Entwicklung zweierlei. Einerseits zeichnet sich eine Verfestigung des mittleren schulberuflichen Ausbildungsweges mit all seinen Strukturnachteilen gegenüber dem Lehrlingsausbildungsprinzip einschließlich einer Dequalifizierungstendenz durch Kurzzeit-Ausbildungen ab. Diese Pfadabhängigkeit wird besonders deutlich an dem relativ jungen Berufsfeld Altenpflege, das wesentlich durch die Verabschiedung des Pflegeversicherungsgesetzes 1994, mit dem das Pflegerisiko erstmals abgesichert wurde, strukturiert ist. Das Gesetz trägt im Geiste des Subsidiaritätsprinzips dem hohen Anteil häuslicher Pflege Rechnung und stellt ambulante vor stationäre Pflege. Es beförderte damit zugleich die Entwicklung beruflicher 
Pflege als Ergänzung zu informeller Pflegetätigkeit, erkennbar nicht zuletzt an der (erst) 2003 verabschiedeten bundeseinheitlichen Regelung der Pflegeausbildung. Sie sieht in Analogie zur Krankenpflege eine schulische Ausbildung mit der Möglichkeit länderspezifischer Regelungen für Kurzzeitausbildungen vor. Auch im Berufsfeld Erziehung und Kinderbetreuung findet sich eine Bestärkung des traditionellen Weges sozialpolitisch beförderter Semi-Professionalität einschließlich einer Fort- und Neuschreibung geschlechtsspezifischer Konnotationen. Nicht nur wird auch in diesem Feld an den variablen Mustern von kürzeren und längeren beruflichen Ausbildungen festgehalten. Das im Oktober 2008 verabschiedete Bundesgesetz zum Ausbau der Betreuung für Kleinkinder, das bis 2013 700.000 Plätze in öffentlicher Betreuung vorsieht, fördert vielmehr neben dem beruflich geprägten Feld der Kinderkrippen auch den Ausbau der häuslichen Tagespflege, wo 30\% der Plätze vorgesehen sind. Dies wird voraussichtlich zu einer erheblichen Erhöhung der Zahl von Tagesmüttern auf über 100.000 führen. Für deren Qualifizierung sind bisher allenfalls Mini-Fortbildungen (wie etwa 100 Stunden Kurszeit in Bayern oder 120 Stunden in Baden-Württemberg) vorgesehen, die deutlich unter den vom Deutschen Jugendinstitut DJI erstellten Musterlehrplan-Volumen von 160 Stunden liegen. Ob angesichts der Finanznot der Kommunen, für die allein aus Kostengründen der Ausbau der Tagespflege attraktiver sein wird als ein Ausbau von Krippen, die mit dem Gesetz auch angestrebte qualitative Verbesserung der Kleinkindbetreuung erreicht werden kann, muss daher als offen angesehen werden. Folgt man der Expertise des Deutschen Jugendinstituts so hängt die dringend benötigte Professionalisierung in der Tagespflege an dem Erfordernis einer soliden Ausbildung und angemessenen Entlohnung; beides ist bereits gegenwärtig nur begrenzt der Fall (Süddeutsche Zeitung Nr. 238 vom 13.10.2008, 10).

Andererseits ist in Pflege und Erziehung ähnlich wie schon ein paar Jahrzehnte vorher im Bereich der Sozialen Arbeit/Sozialpädagogik inzwischen durchaus eine allmähliche Professionalisierung erkennbar: mit der für Deutschland neuen Akademisierung der Pflege und der Entdeckung der Frühpädagogik als wissenschaftlicher Disziplin. In der Pflege sind seit den neunziger Jahren des letzten Jahrhunderts sukzessive vornehmlich an Fachhochschulen neue Studiengänge für Pflegemanagement und Lehr- und Weiterbildungstätigkeit in der Pflege entstanden. Freilich zeigt sich auch hier eine im Vergleich zu den angloamerikanischen Vorbildern zeitlich deutlich verzögerte Akademisierung und bis heute auch eher geringe Verwissenschaftlichung: So bleibt die Pflegeausbildung selbst als Berufsausbildung unterhalb des akademischen Niveaus und für die meisten Studiengänge wird Praxiserfahrung in der Pflege als Zulassungsvoraussetzung gefordert. Die Zahl der Studiengänge an Universitäten ist klein; eine Ausbildung für Pflegeforschung findet kaum statt und eine entsprechende wissenschaftliche Infrastruktur ist bisher nur gering entwickelt (Sewtz 2006).

\section{$5 \quad$ Ausblick: Grenzen und Potenziale sozialpolitischer Regulierung}

Die letztgenannten Beispiele sozialpolitischer Reformen zeigen, dass der gestiegene Bedarf an qualifizierten Dienstleistungen in den Bereichen Erziehung und Pflege gesellschaftlich und politisch durchaus anerkannt wird. Soziale Dienstleistungen expandieren und finden als informelle Arbeit durch die finanzielle Unterstützung häuslicher Pflege und familiennaher Kinderbetreuung stärkere Anerkennung. Eine echte Professionalisierung 
findet freilich nur mehr auf höherem Niveau und auch hier erst in Ansätzen statt. Für den großen Kernbereich verberuflichter Arbeit zeichnet sich gleichzeitig nicht nur eine Fortschreibung der Defizite schulberuflicher Ausbildung sondern durch Kurzzeitausbildungen und Niedriglohnbeschäftigung auch eine verstärkte Polarisierung von Erwerbsstrukturen ab. Dies hängt auch damit zusammen, dass der mittlere Bereich gegenwärtig kaum von der angeführten Akademisierung profitieren kann, weil das Potenzial für Professionalisierung durch die in den Gesundheits- und Erziehungsberufen schon immer vorhandenen und gegenwärtig erneut sozialpolitisch gestützten offenen Flanken zur informellen Arbeit ein Stück weit konterkariert wird. In diesem Kontext erweist sich der fehlende Qualifikationsschutz der vollzeitschulischen Ausbildungen im Sozialbereich als Einfallstor für gering qualifizierte Arbeit, wie die Expansion der Kurzzeitausbildungen zeigt. Diese Entwicklung ist auch deshalb problematisch, weil sie gegenwärtig durch das Verhalten von Wohlfahrtsverbänden und Kommunen als den dominanten Arbeitgebern im Feld sozialer Dienstleistungen forciert wird. Denn mit der Einführung von organisiertem Wettbewerb im Sozialsektor findet eine Ökonomisierung der Erbringung sozialer Dienstleistungen statt, die statt Professionalisierung eher Niedriglohnbeschäftigung befördert (Kühnlein/ Wohlfahrt 2006). So ist der Bereich stationärer Altenpflege, in dem private und NonProfit-Anbieter konkurrieren, vielerorts zum Niedriglohnsektor geworden, für den die Verbände der freien Wohlfahrtspflege, die sich nicht mehr in der Lage sehen, Tariflöhne zu zahlen, nunmehr einen Mindestlohn fordern (vgl. für Niedersachsen: Weserkurier Nr. 267, 12.11.2008, 2).

Gleichzeitig steigen die Ansprüche an die Qualität der sozialen Dienstleistungen, wie man an der aktuellen Diskussion zur Notwendigkeit bildungsorientierter frühpädagogischer Förderung in der Kinderbetreuung sieht. Diese Qualitätsansprüche stehen nicht nur im Kontrast zu traditionellen pädagogischen Betreuungskonzepten und Ausbildungsstrukturen sondern auch den Arbeitsbedingungen und Entlohnungen im Erzieherberuf. Der Beruf ermöglicht einer vollzeitbeschäftigten Erzieherin, die in der Regel eine vier- bis fünfjährige fachschulische Ausbildung absolviert hat, selbst bei mehrjähriger Berufserfahrung kaum eine Existenzsicherung. Er gilt neben dem geringen Einkommen auch angesichts hoher Gruppenstärken in den Kinderbetreuungseinrichtungen und geringer Aufstiegsmöglichkeiten als so wenig attraktiv, dass bereits gegenwärtig vor allem Großstädte angesichts des geplanten Ausbaus der Kleinkinderbetreuung mit Arbeitskräftemangel rechnen (Süddeutsche Zeitung Nr. 261, 10. 11.2008, 9).

Dass soziale Dienstleistungen durchaus auch attraktive Beschäftigungsfelder sein können, zeigt im Bereich ,Erziehung und Bildung' Frankreich, wo der republikanische Staat einheitliche Professionalisierungswege für die Erziehung von Kindern und jungen Menschen geschaffen hat oder im Bereich der Pflege Schweden, wo, ausgehend von dem Leitbild der Pflege als Bürgerrecht, Pflegetätigkeit als qualifizierter Beruf befördert wird (Theobald 2008). Selbst in den marktliberalen angloamerikanischen Ländern konnten sich im Gesundheitssektor einzelne Berufsgruppen durchaus erfolgreich gegen SemiProfessionalität zur Wehr setzen (Kuhlmann 2006). Für Deutschland mag angesichts des historischen Erbes weder eine, Verstaatlichung' fürsorglicher Tätigkeit noch die Strategie von ,professional projects' in offenen Märkten eine Lösung sein, denn Letzteres bedeutet neben der weitgehenden Akademisierung sozialer Dienstleistungen als Kehrseite auch, dass die qualitativ hochwertigen Erziehungs- und Gesundheitsdienstleistungen nur den kaufkräftigen sozialen Gruppen zugänglich sind. Auch ändern die steigenden Bedarfe 
an sozialen Dienstleistungen nichts daran, dass eine weitgehende Formalisierung der ,Arbeit am und mit Menschen' wenig wünschenswert erscheint (Senghaas-Knobloch 2008; Saraceno 2008).

Gleichwohl schließen gesellschaftlich akzeptable Bedingungen für ,care giving' und ,care receiving' die Notwendigkeit ein, diese Tätigkeiten neben der Anerkennung unbezahlter und ehrenamtlicher Arbeit auch als berufliche Arbeit so zu strukturieren, dass sie den Standards von Normalarbeit und Geschlechtergerechtigkeit entspricht. Dafür wären neben dem Ausbau der bisher nur zögerlich erfolgten Verwissenschaftlichung in den Bereichen Frühpädagogik und Pflege eine Qualitätssicherung und Vereinheitlichung in den dreijährigen beruflichen Ausbildungen notwendig, die berufliche Entwicklungsfähigkeit über kontinuierliche Weiterbildung und Eröffnung von Wegen in die akademische Ausbildung sichern müssten. Dies setzt akademisch qualifiziertes Ausbildungspersonal ebenso voraus wie eine Gestaltung der Arbeits- und Entlohnungsbedingungen in den entsprechenden Berufen, die sich an den Standards von Normalarbeit orientiert. Notwendig ist weiterhin ein gesellschaftlicher Konsens darüber, dass ,gute Erziehung' und ,gute Pflege' menschenwürdige Arbeitsbedingungen voraussetzen und hier, wie in anderen Erwerbsbereichen auch, eine arbeitspolitische Regulierung gefordert ist. Anerkannt werden müsste auch, was etwa bei ärztlichen oder juristischen Dienstleistungen ebenso wie bei guter handwerklicher Arbeit selbstverständlich ist: dass qualitativ hochwertige soziale Dienstleistungen nicht zum Nulltarif zu haben sind. Die eigentliche Herausforderung für den deutschen Wohlfahrtsstaat besteht insofern weniger darin, den Mix aus familialer, ehrenamtlicher und beruflicher Tätigkeit aufzukündigen als vielmehr darin, Fürsorge und Erziehungstätigkeit als eine unabweisbare Lebensnotwendigkeit und gesellschaftlich wertgeschätzte Arbeit auch in Form einer nachhaltigen Beruflichkeit zu ermöglichen.

\section{Literatur}

Achatz, Juliane (2005): Geschlechtersegregation im Arbeitsmarkt; in: Martin Abraham, Thomas Hinz (Hg.): Arbeitsmarktsoziologie. Probleme, Theorien, Empirische Befunde. Wiesbaden, 263-301

Autorengruppe Bildungsberichterstattung (Hg.) (2008): Bildung in Deutschland 2008. Ein indikatorengestützter Bericht mit einer Analyse zu Übergängen im Anschluss an den Sekundarbereich I. Bielefeld

Backes, Gertrud M., Ludwig Amrhein, Martina Wolfinger (2008): Gender in der Pflege. Herausforderungen für die Politik. Expertise im Auftrag der Friedrich-Ebert-Stiftung. Bonn

Baethge, Martin (1999): Glanz und Elend des deutschen Korporatismus in der Berufsbildung; in: WSI-Mitteilungen 52, 8, 489-497

Baethge, Martin, Heike Solga, Markus Wieck (2007): Berufsbildung im Umbruch. Signale eines überfälligen Aufbruchs. Studie der Friedrich-Ebert-Stiftung. Berlin

Berger, Johannes, Claus Offe (1984): Die Entwicklungsdynamik des Dienstleistungssektors; in: Claus Offe (Hg.): Arbeitsgesellschaft: Strukturprobleme und Zukunftsperspektiven. Frankfurt a.M./New York, 229-270

Bothfeld, Silke u.a. (2005): WSI-FrauenDatenReport 2005. Handbuch zur wirtschaftlichen und sozialen Situation von Frauen. Berlin

Bundesknappschaft Minijob-Zentrale (2004): Aktuelle Entwicklungen im Bereich der geringfügigen Beschäftigungen. 3/2004. Essen 
Deutsche Rentenversicherung Knappschaft Bahn-See/ Minijob-Zentrale (2007): Aktuelle Entwicklungen im Bereich der geringfügigen Beschäftigung. IV. Quartal 2007. Essen

Dingeldey, Irene (2006): „Holistic Governance“ oder die Notwendigkeit reflexiver Gestaltung von Familien- und Arbeitsmarktpolitik. Zur differenten Entwicklung der Vereinbarkeit von Familie und Beruf in Dänemark, Großbritannien und der Bundesrepublik; in: Hans Bertram, Helga Krüger, C. Katharina Spieß (Hg.): Wem gehört die Familie der Zukunft? Expertisen zum 7. Familienbericht der Bundesregierung. Opladen, 359-381

Europäische Kommission (2006): Umsetzung des Gemeinschaftsprogramms von Lissabon. Die Sozialdienstleistungen von allgemeinem Interesse in der Europäischen Union. Luxemburg

Evers, Adalbert, Christoph Sachße (2003): Social Care Services for Children and Older People in Germany: Distinct and Separate Histories; in: Anneli Anttonen, John Baldock, Jorma Sipilä (Hg.): The Young, the Old and the State. Cheltenham, 55-80

Gottschall, Karin (2001): Zwischen tertiärer Krise und tertiärer Zivilisation. Zur sozialwissenschaftlichen Analyse von Dienstleistungsgesellschaften; in: Berliner Journal für Soziologie $11,2,217-235$

Gottschall, Karin (2004): Vom Statuserhalt zur Sozialinvestition? Erziehung und Bildung als Sozialstaatstransformation; in: Zeitschrift für Sozialreform 50, 1-2, 126-147

Gottschall, Karin, Karen Hagemann (2002): Die Halbtagsschule in Deutschland: Ein Sonderfall in Europa?; in: Aus Politik und Zeitgeschichte, Beilage zur Wochenzeitschrift „Das Parlament“ B41, 12-22

Hinrichs, Karl (1996): Das Normalarbeitsverhältnis und der männliche Familienernährer als Leitbilder der Sozialpolitik; in: Sozialer Fortschritt 4, 102-107

Kalina, Thorsten, Claudia Weinkopf (2006): Mindestens sechs Millionen Niedriglohnbeschäftigte in Deutschland: Welche Rolle spielen Teilzeitarbeit und Minijobs? IAT-Report 2006-03. Gelsenkirchen (http://www.iaq.uni-due.de/iat-report/2006/report2006-03.pdf)

Krüger, Helga (1995): Prozessuale Ungleichheit. Geschlecht und Institutionenverknüpfungen im Lebenslauf; in: Peter A. Berger, Peter Sopp (Hg.): Sozialstruktur und Lebenslauf. Opladen, 133-153

Krüger, Helga (2003). Berufliche Bildung. Der deutsche Sonderweg in der Geschlechterfrage; in: Berliner Journal für Soziologie 13, 4, 497-510

Kuhlmann, Ellen (2006): Modernising Health Care. Reinventing professions, the state and the public. Bristol

Kühnlein, Gertrud, Norbert Wohlfahrt (2006): Soziale Träger auf Niedriglohnkurs? - Zur aktuellen Entwicklung der Arbeits- und Beschäftigungsbedingungen im Sozialsektor; in: WSI-Mitteilungen 59, 7, 389-395

Leibfried, Stephan, Uwe Wagschal (Hg.) (2000): Der deutsche Sozialstaat. Bilanzen - Reformen - Perspektiven. Frankfurt/New York

Nagel, Bernhard (2000): Der Erzieherberuf in seiner historischen Entwicklung; in: Bildung, Erziehung, Betreuung von Kindern in Bayern, 5, 1, 11-13

OECD (2007): PISA 2006. Science Competencies for Tomorrow's World. Paris

Paulini, Christa (2007): Soziale Arbeit. Vom Eignungsberuf für Frauen zum Beruf für genderbewusste Fachkräfte-logische Konsequenz oder Utopie?; in: Elke Kruse, Evelyn Tegeler (Hg.): Weibliche und männliche Entwürfe des Sozialen. Wohlfahrtsgeschichte im Spiegel der Genderforschung. Opladen/Farmington Hills, 73-87

Saraceno, Chiara (2008): „Care“ leisten und „Care“ erhalten zwischen Individualisierung und Refamilialisierung; in: Berliner Journal für Soziologie 18, 2, 244-256

Senghaas-Knobloch, Eva (2008): Care-Arbeit und das Ethos fürsorglicher Praxis unter neuen Marktbedingungen am Beispiel der Pflegepraxis; in: Berliner Journal für Soziologie 18, 2, 221-243

Sewtz, Susanne (2006): Karrieren im Gesundheitswesen. Eine geschlechtervergleichende Analyse der Professionen Medizin und Pflege. Weinheim/München

SOFI (Soziologisches Forschungsinstitut) u.a. (Hg.) (2005): Berichterstattung zur sozioökonomischen Entwicklung in Deutschland. Arbeit und Lebensweisen. Erster Bericht. Wiesbaden 
StBA (Statistisches Bundesamt) (1999): Fachserie 11 Reihe 2. Bildung und Kultur. Berufliche Schulen. Schuljahr 1998/1999. Wiesbaden

StBA (Statistisches Bundesamt) (2003): Fachserie 11 Reihe 2. Bildung und Kultur. Berufliche Schulen. Schuljahr 2002/2003. Wiesbaden

StBA (Statistisches Bundesamt) (2005): Bevölkerung und Erwerbstätigkeit. Beruf, Ausbildung und Arbeitsbedingungen der Erwerbstätigen. Ergebnisse des Mikrozensus 2003, Bd. 2: Deutschland. Fachserie 1, Reihe 4.1.2. Wiesbaden

StBA (Statistisches Bundesamt) (2007): Fachserie 11 Reihe 2. Bildung und Kultur. Berufliche Schulen. Schuljahr 2006/2007. Wiesbaden

StBA (Statistisches Bundesamt) (2008): Mikrozensus. Bevölkerung und Erwerbstätigkeit. Beruf, Ausbildung und Arbeitsbedingungen der Erwerbstätigen. Bd. 2: Deutschland. Fachserie 1, Reihe 4.1.2. Wiesbaden

Theobald, Hildegard (2008): Care-Politiken, Care-Arbeitsmarkt und Ungleichheit: Schweden, Deutschland und Italien im Vergleich; in: Berliner Journal für Soziologie 18, 2, 257-281

Voß, G. Günter, Hans J. Pongratz (1998): Der Arbeitskraftunternehmer - Eine neue Grundform der Ware Arbeitskraft?; in: Kölner Zeitschrift für Soziologie und Sozialpsychologie 50, 1, $131-158$

Anschrift der Verfasserin:

Prof. Dr. Karin Gottschall

Universität Bremen

Zentrum für Sozialpolitik

Abt. Geschlechterpolitik im Wohlfahrtsstaat

Parkallee 39

28209 Bremen

E-Mail: k.gottschall@zes.uni-bremen.de

Schlagwörter: Frauenarbeit, öffentliche Arbeitgeber, Professionalisierung, Sozialpolitik, soziale Dienstleistungen 